\title{
EchoGéo
}

$47 \mid 2019$

Nouvelles géographies de la collecte

\section{Du glanage et de la géographie}

Entretien de Rémi de Bercegol avec Mathias Faurie, réalisé le 3 avril 2019.

\section{Mathias Faurie et Rémi de Bercegol}

\section{OpenEdition}

\section{Journals}

Édition électronique

URL : https://journals.openedition.org/echogeo/17282

DOI : $10.4000 /$ echogeo. 17282

ISSN : 1963-1197

Éditeur

Pôle de recherche pour l'organisation et la diffusion de l'information géographique (CNRS UMR 8586)

Référence électronique

Mathias Faurie et Rémi de Bercegol, « Du glanage et de la géographie », EchoGéo [En ligne], 47 | 2019, mis en ligne le 21 avril 2019, consulté le 10 août 2021. URL : http://journals.openedition.org/echogeo/ 17282 ; DOI : https://doi.org/10.4000/echogeo.17282

Ce document a été généré automatiquement le 10 août 2021.

EchoGéo est mis à disposition selon les termes de la licence Creative Commons Attribution - Pas d'Utilisation Commerciale - Pas de Modification 4.0 International (CC BY-NC-ND) 


\section{Du glanage et de la géographie}

Entretien de Rémi de Bercegol avec Mathias Faurie, réalisé le 3 avril 2019.

\section{Mathias Faurie et Rémi de Bercegol}

1 Après des études de géographie à l'Université Paris 1 Sorbonne, Mathias Faurie soutient un doctorat au sein du laboratoire PRODIG en 2011 qui le fait voyager jusqu'en Nouvelle-Calédonie. Glaneur né, il finance alors déjà une partie de ses études par la vente de truffes qu'il récupère lors de ses pérégrinations dans les forêts de Bourgogne. Après ses études, il travaille en tant qu'ATER à l'Université Paris 4 puis à l'Université d'Angers. Il effectue un post-doctorat à l'IRD et au MNHN. En parallèle, Mathias Faurie mûrit depuis longtemps un projet d'installation en Dordogne grâce à un modeste terrain qui lui permet de concilier ses passions pour le glanage, la pêche... et les pommiers. En 2018, il finit par suivre une formation pour professionnaliser sa petite exploitation de pommes et autres fruits du verger. Aujourd'hui artisan cidrier, il vient d'embouteiller deux mille litres de jus de pomme et 4000 litres de cidre. De la ville à la campagne, son métier et son vécu éclairent la pratique et la mise en pratique de la géographie au quotidien.

Rémi de Bercegol (RdB). Bonjour Mathias Faurie, bienvenue dans la Rubrique Sur le métier d'EchoGéo. Nous voulions recueillir votre témoignage de géographe, de glaneur, et de cidriculteur, mais avant toute chose, pourriez-vous rapidement nous présenter votre métier aujourd'hui ? Est-il juste de vous présenter comme un cidriculteur?

Mathias Faurie (MF). Oui, tout à fait, ma principale activité est celle de cidriculteur, à Molières où j'exploite un verger de 2 hectares, en Dordogne. Je produis du jus de pomme et du cidre, que je commercialise sous la marque " Maison Rouge », du nom de ma maison située au lieu-dit Caminade. Le nom " Maison Rouge » était celui de la ferme de mes arrière-grands-parents, que je me suis appliqué à restaurer en parallèle de mes années de thèse. Je développe aussi depuis peu deux autres types de cidres en petites bouteilles : un "Sydra du sud-ouest » et un «CidrOnoix du Périgord ». Enfin, outre le cidre, je produis aussi des truffes du Périgord, des noix, du raisin et d'autres fruits, ainsi que des légumes pour mon autoconsommation. 
(RdB). Vous avez choisi de vous investir dans un métier assez atypique pour un géographe de formation, pourriez-vous nous expliquer les raisons de cette conversion?

(MF). J'ai suivi un cursus de géographie à l'Université Paris 1 - Panthéon-Sorbonne, initialement attiré par l'option de documentaire vidéo. Cette formation, intéressante, me laissait insatisfait par son aspect 'géographie de cabinet' alors que c'est bien une géographie de terrain qui m'avait attiré ...et que j’ai pratiqué par moi-même... hors des bancs de l'université.

J'ai eu la "bougeotte" tout au long de mes études. J'ai sillonné les campagnes. J'y ai découvert un monde rural singulier, des territoires presque vides d'hommes, et finalement un exotisme semblable à certains lieux reculés de la Terre. L'exode rural a laissé un monde en friche, un passé agricole enseveli. La monoculture d'une part et l'enfrichement d'autre part ont quasiment rendu invisible la polyculture d'avantguerre. C'est dans ces décombres que j'ai pris conscience de la richesse que représentent ces derniers reliquats : vignes familiales abandonnées, vergers oubliés, variétés fruitières presque disparues.

(RdB). C'est aussi durant vos études que vous avez commencé à glaner ici et là ?

(MF). Pas tout à fait: le glanage, je le tenais en réalité déjà de mes parents qui glanaient à la sortie du marché pour nourrir nos poules, lapins, chats et chien. Avoir des animaux, c'est savoir que rien ne se perd et que tout se récupère. Ensuite, durant mes études de géographie et mes terrains de recherche dans l'hémisphère Sud, et en particulier en Océanie, j'ai été pris du virus de la cueillette, des prélèvements de boutures et graines sauvages recueillies puis testées au jardin, aux emprunts de variétés faites chez les uns chez les autres : le glanage est pour les sociétés un moyen puissant de sélection et de diversification génétique.

Savoir glaner dans la nature, connaître les variétés sauvages ou semi-cultivées, c'est se prévenir des disettes : le glanage est un savoir qui permet la résilience des sociétés suite aux crises et aux aléas, que ceux-ci soient d'origines humaines ou naturelles

$(R d B)$. Il paraît que vous financiez alors une partie de vos études par la vente de truffes glanées à l'école buissonnière. En quoi cette expérience de collecte a-t-elle été fondatrice de votre activité ?

(MF). C'est vrai. En parcourant les campagnes reculées, je me suis rendu compte que les truffes avaient pour terrain de prédilection ces vignes et vergers oubliés, du fait de l'abandon de l'entretien et du pastoralisme. Ce champignon, que ce soit la truffe de Bourgogne ou la truffe du Périgord, aime les milieux semi-ouverts en voie de reconquête par les arbres. Très vite, l'aspect pécuniaire tiré de la vente des truffes récoltées à des restaurants, est passé au second plan, et l'amusement lié à l'exploration a pris le dessus. J'ai adopté un chien, qui était fou de truffes, et a découvert cela en même temps que moi... Des fois, je ne sais plus trop s'il m'a montré la voie ou bien si c'est moi qui le guidais!

Illustration 1 - Une collecte réussie

Une poignée de truffes glanée dans une friche agricole

EchoGéo, 47 | 2019 


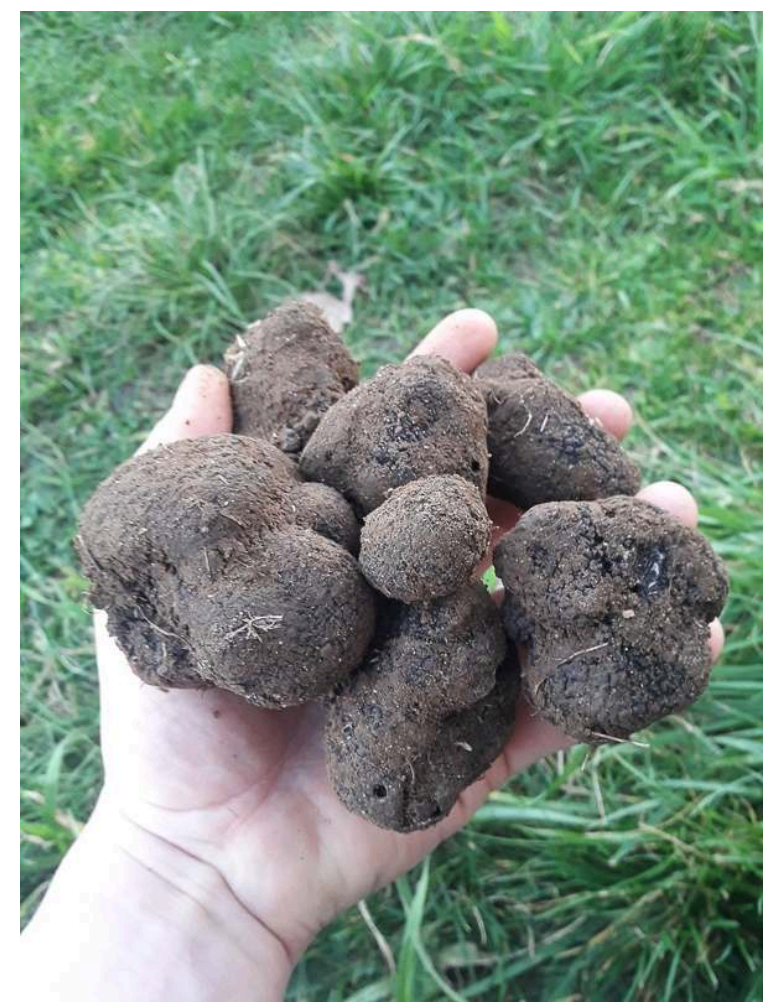

Auteur: M. Faurie, 2018.

2 Après quelques repérages sur Géo-portail, nous faisions des prospections, des marquages en mettant en pratique les outils de la géographie pour repérer et appréhender les parcelles et les terroirs oubliés. Je regardais les cadastres, la toponymie. Je tentais ainsi de comprendre des logiques et géo-référençait les lieux sur les cartes topographiques. J'étudiais les formations et associations végétales. Je potassais les cartes géologiques pour comprendre les terroirs: sols, expositions, anciennes activités agropastorales. 
Illustration 2 - Anciennes vignes, nouvelles truffières... !

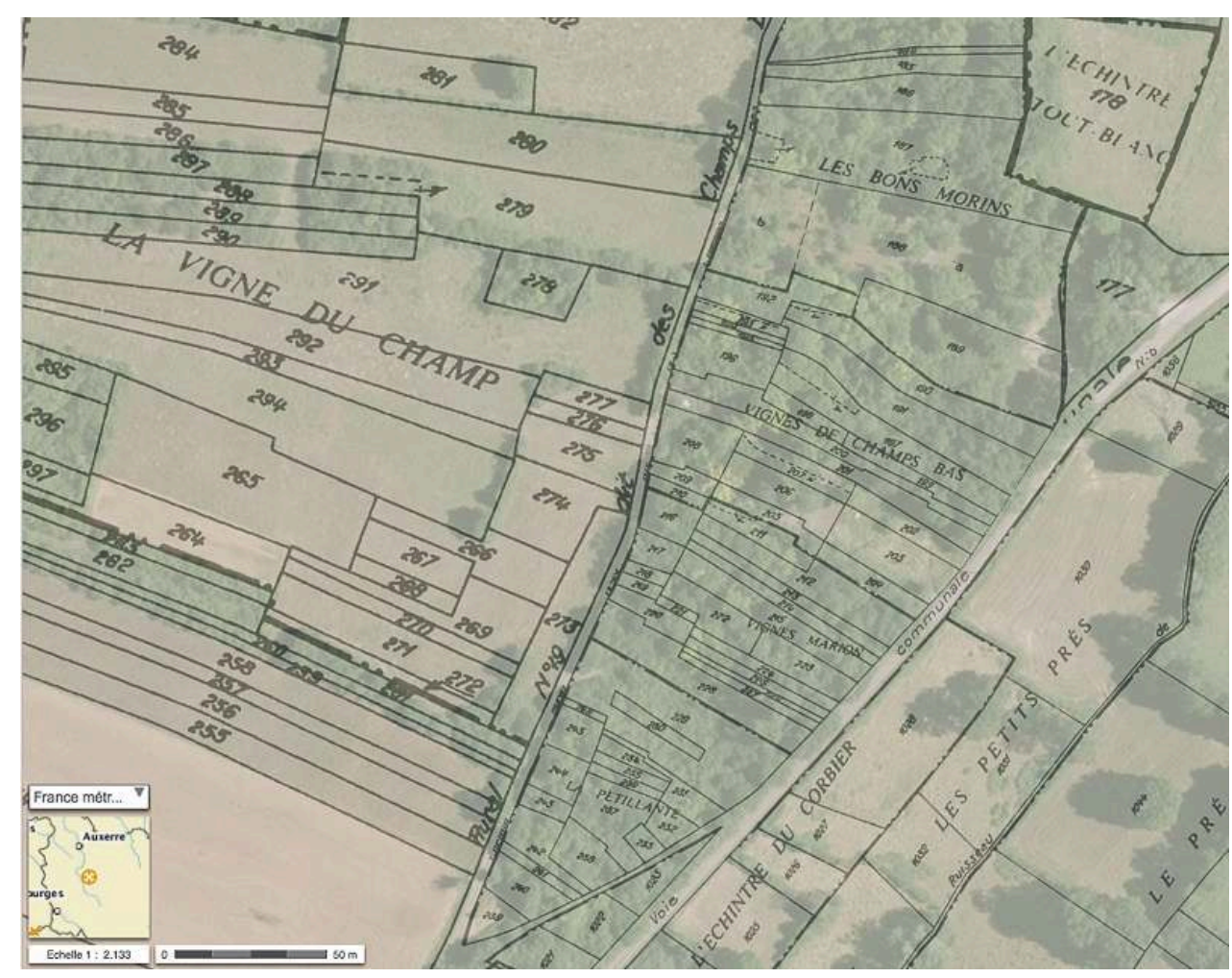

Extrait de l'ancien cadastre et de la BD Ortho de I'IGN sur Géoportail. Superposition du registre cadastral et d'une vue aérienne en Bourgogne, près de Vézelay (Yonne)

Source : Géoportail. Auteur : M. Faurie, 2016.

(RdB). Et c'est donc à ce moment-là que vous avez commencé à élargir l'éventail de votre collecte.

(MF). Peu à peu, l'idée m'est venue de récolter au-delà des truffes, en glanant des champignons, des fruits, et notamment différentes variétés de raisins et de pommes. J'ai commencé à transformer les raisins glanés en " piquette ", à broyer et presser les pommes pour en tirer des cidres. J'effectuais des prélèvements de variétés pour constituer mes propres vergers. Le cidre que je produis aujourd'hui est en grande majorité issu de ce glanage.

Les pommes se sont avérées être les fruits les plus faciles pour assouvir ma passion compulsive de glanage : elles sont disponibles en masse, les pommiers oubliés et abandonnés ne se comptent plus à travers les campagnes. Les vignes abandonnées sont intéressantes mais moins courantes et les milieux se refermant vite, leur durée d'existence est de courte durée. 
Illustration 3 - Pommes glanées

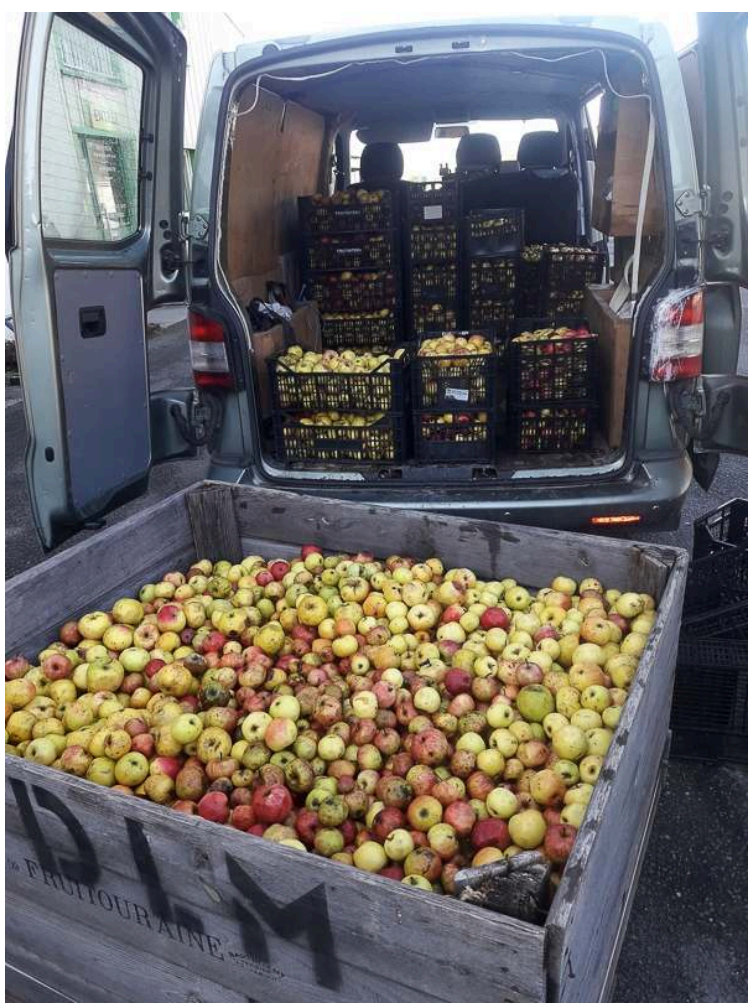

Une dizaine de variétés de pommes anciennes issues d'un verger à l'abandon Auteur: M. Faurie, 2018

De plus, j'ai perçu que le jus de pomme et le cidre allaient connaître un renouveau : les jus d'orange et autres multivitaminés à base de fruits industriels et concentrés sont de moins en moins à la mode ; et le cidre peut bénéficier indirectement de l'élan mondial pour les vins naturels d'une part et de l'essor des microbrasseries d'autre part. Le cidre est un produit de terroir oublié, perverti par son industrialisation. Les variétés anciennes sont en voie de disparition, alors qu'elles sont peut-être notre patrimoine de demain. La diversité fera partie des solutions permettant la résilience des sociétés et un impact moins destructeur sur les écosystèmes. Les savoirs vernaculaires et scientifiques peuvent être réunis pour une meilleure efficience.

Mon objectif d'exploitation est aussi d'avoir une portée conservatoire, la glane m'ayant permis de constituer une belle collection spécifique et variétale

$(\mathrm{RdB})$. Le glanage est donc bien une partie essentielle de votre métier de cidriculteur. Quel regard portez-vous sur votre pratique et les lieux dans lesquels elle s'insère?

(MF). Je produis aujourd'hui du cidre, mais je reste un glaneur.

Le glanage que je pratique n'est plus celui d'un temps où l'on trouvait des dates officielles de glanage dans les champs et dans les vignes, après les moissons, ou après les dates de clôtures des vendanges. Il s'agit plutôt de prélèvements ponctuels dans un monde rural délaissé, où les familles et les paysans disparaissent, où les villages se vident de leurs habitants, et où, en semaine un mois de novembre, c'est l'humanité tout entière qui semblerait presque avoir disparu...

Aujourd'hui, à l'image de ce monde abandonné, seuls quelques cépages de raisins et quelques variétés de fruits sont encore cultivés, et certaines s'éteignent alors qu'elles 
sont là, à portée de main et que personne ne les ramasse car il n'y a justement personne pour les ramasser. Je me suis juste retrouvé dans un monde où le glanage de fruits est finalement plus facile qu'auparavant.

Si vous saviez...il y a tellement de fruits non ramassés...notamment de pommes et de poires, provenant de vieux arbres, ceux qui donnent les meilleurs fruits et les meilleurs jus... Leurs racines sont au contact du sous-sol et le terroir s'exprime pleinement dans leur production. Je n'ai de cesse de trouver de nouveaux vergers, je rencontre les descendants des personnes qui les ont plantés et qui sont restés. S'ils ne font rien des fruits je leur propose de les récolter en échange de cidre ou de jus de pomme, voire d'un petit billet s'ils préfèrent.

$(\mathrm{RdB})$. Au-delà de ce glanage, en quoi consiste concrètement votre activité quotidienne de cidriculteur? Pourriez-vous nous en dire un peu plus sur votre exploitation?

(MF). Aujourd'hui mon exploitation mesure 2 hectares, et j'ai pour projet d'arriver à 5 à 6 hectares dont 3,5 hectares de pommiers. C'est une exploitation de taille a priori réduite quand on la met en regard des pratiques dominantes en agriculture. Comme je vous le disais auparavant, le cidre est une boisson qui a bien trop souffert de l'industrialisation de l'agroalimentaire. Il est devenu un produit issu de la monoculture de quelques variétés de pommes, issu de jus concentré, modifié par des intrants chimiques, puis pasteurisé, comme la plupart des cidres que l'on peut trouver en grande distribution. L'image de ce produit est ternie par les mauvaises pratiques, et les cidres de terroir ont presque disparu.

Vous l'avez compris, j'essaye donc de développer une autre pratique, à contrecourant de ce système. Mes cidres sont pur jus, mes fermentations sont lentes et menées avec des levures indigènes, la prise de mousse se fait naturellement en bouteille. Mon cidre est un produit vivant, et cela fait sens pour moi. Je produis et transforme à la ferme. Mon métier est donc celui d'un travail au verger. Je greffe mes arbres fruitiers moi-même et les élève avant de les planter, je conduis les arbres en formes naturelles, je ne pratique que de rares interventions biologiques. 


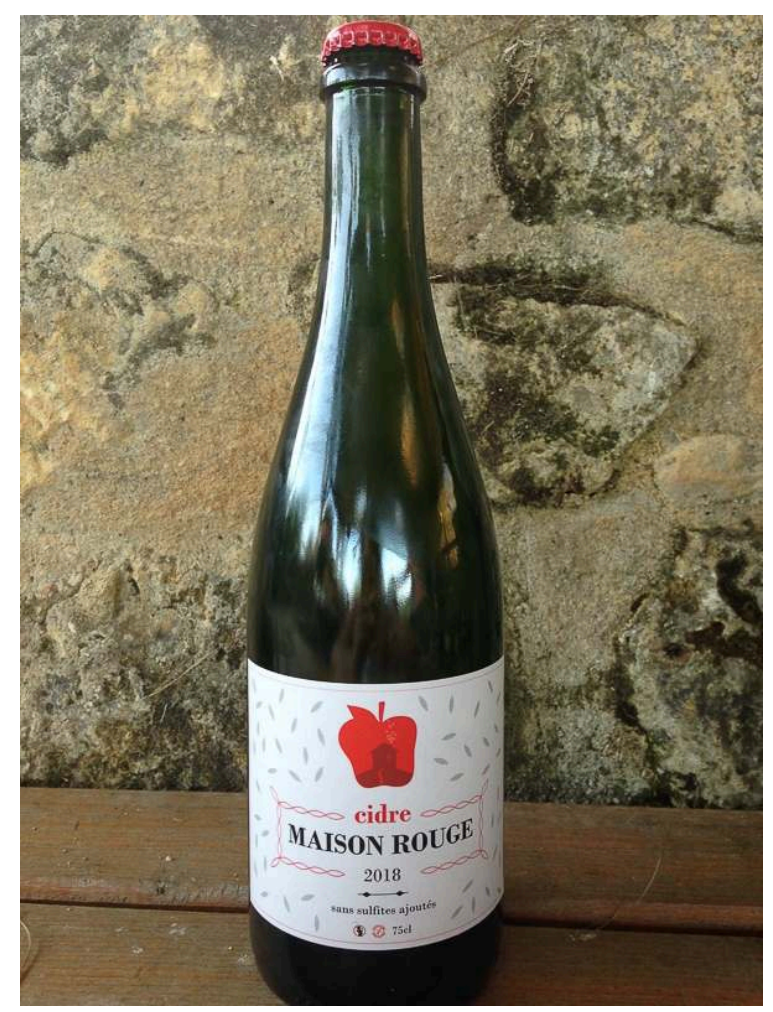

Auteur : M. Faurie.

Je fais tout à la main et il y a de la pénibilité, c'est sûr... mais peu à peu je compte trouver des solutions pour rendre le travail moins difficile et soulager mon dos pour ce qui concerne les charges lourdes. Au verger, l'effort physique me plaît beaucoup, je suis au contact des éléments. Il y a bien sûr la partie administrative et comptable, qui me plait un peu moins alors qu'elle est étonnamment prenante en agriculture, notamment pour obtenir la certification biologique, et encore plus lorsqu'il s'agit de la vente de boissons alcoolisées.

Mes journées sont en tout cas bien remplies car le métier de cidriculteur se fait à la fois au verger pour la production, à la cave et à l'atelier pour la transformation, et sur les routes de France pour la commercialisation.

(RdB). Que pensez-vous justement du label biologique que vous venez d'évoquer? Pour nombre de consommateurs, il apparaît comme un gage de qualité.

3 (MF). Au-delà de ce processus de certification biologique et des débats qui l'entourent, il me semble pour ma part urgent de désindustrialiser l'exploitation du monde du vivant. Aujourd'hui, l'objectif n'est plus de nourrir les Européens à la sortie de la guerre mais de produire mieux pour le bonheur et la santé de tous, en préservant l'environnement et en faisant face intelligemment aux évolutions bioclimatiques. Le défi le plus crucial selon moi réside dans une remise en question des produits phytosanitaires afin de mettre en place une agroécologie respectueuse de son environnement.

4 Le maraîchage est bien souvent mis en avant en agroécologie mais pour ce qui concerne les grandes cultures, qui permettent aussi et surtout de nourrir l'humanité, notamment dans les pays en développement (riz, maïs, blé, soja, alimentation animale...), on 
manque de performances scientifiques et d'incitations politiques. En ce sens, le label biologique est une première base garantissant des pratiques respectueuses de l'environnement et le bien-être animal. On peut faire encore mieux et pousser la démarche plus loin, mais c'est déjà un premier pas, malgré les récupérations commerciales qu'il induit.

(RdB). Les populations urbaines imaginent souvent les campagnes vidées de leurs jeunes actifs. L'image est-elle juste? Vous êtes la preuve du contraire et j'imagine que comme vous, d'autres choisissent de s'installer loin des agglomérations urbaines.

(MF). Il est incontestable qu'on assiste à un retour dans les zones rurales d'une population urbaine et plus ou moins jeune. Mais ce ne sont pas tout à fait les mêmes paradigmes que dans les années 1960-1970.

La technologie offre de plus en plus de possibilités et décloisonne les espaces, rend les modes de vies métisses. Une majorité des néoruraux vit dans une transposition du mode de vie urbain, investie dans de nouveaux lieux mais reliée à la ville via la technologie. Je pense qu'on déplace à la campagne son habitation et son centre de gravité tout en restant mobile et connecté. Les zones rurales et les jeunes actifs qui s'y installent ont un grand besoin de la ville pour y effectuer de nombreuses démarches, y accéder aux activités culturelles, mais surtout la ville est bien souvent le débouché de leur production, comme c'est le cas par exemple pour une partie de mon cidre.

En tous cas, je pense que cette néo-ruralité contribue peu à peu à transformer les campagnes. Il y a parfois des incompréhensions entre anciens et nouveaux venus. Mais il y a aussi souvent des alliances inattendues, et cela ressort notamment sur le plan des activités économiques, ou encore lors des élections municipales. On assiste donc à une redéfinition des jeux de pouvoirs dans les petites communes rurales du fait de ces changements de population et d'activités.

$(\mathrm{RdB})$. De la ville à la campagne, de glaneur à cidriculteur en passant par géographe, le fil conducteur de votre existence est intimement lié à la pratique de la terre. Mais votre exploitation n'est-elle pas vouée, par nature, à s'enraciner peu à peu...et par là-même à fixer votre existence?

(MF). Les racines sont comme les branches de l'arbre: elles $n$ 'en finissent pas de pousser.

Être exploitant agricole me permet de m'adonner pleinement à ma passion, mais je ne pense pas que mon itinéraire soit figé. Je ne pense pas que mon parcours en géographie soit achevé, car ces histoires s'entrelacent et les séparations qui peuvent apparaître ont surtout des fondements pratiques et académiques. Je pratique la construction de mon verger et de ma ferme avec un esprit scientifique, et ce que j'apprends chaque jour nourrit mon envie de le transmettre un jour, pourquoi pas sous forme d'enseignements. À travers cette aventure je me suis penché sur une foule de sujets passionnants, j'ai dû retourner en bibliothèque, me procurer des livres et articles très instructifs, j'ai dû m'ouvrir à plusieurs autres disciplines, notamment en science de la vie et de la terre. Mais c'est au plus près du terrain que j'aurais glané la plupart de ces nouveaux savoirs, acquis avec le lancement de mon projet agricole.

Mon objectif est aujourd'hui de poursuivre la construction d'un verger conservatoire et d'un jardin rassemblant des végétaux prélevés ici et là, des pratiques et savoirs glanés au fil du temps et des lieux. Alors bien sûr, en m'installant en tant 
qu'agriculteur et en produisant mes truffes et mes fruits, je glane de moins en moins. Mon premier verger a 8 ans et plusieurs sont en cours d'implantation.

$(R d B)$. Un jour ou l'autre, on viendra donc glaner entre vos pommiers...

(MF). En fait c'est déjà le cas et j'ai vu des plus jeunes me grappiller quelques fruits. Ça me fait sourire... bientôt il y aura tellement de pommes qu'on ne pourra plus les compter!

\section{AUTEURS}

\section{MATHIAS FAURIE}

Mathias Faurie est géographe et artisan cidrier.

\section{RÉMI DE BERCEGOL}

Rémi de Bercegol, remi.debercegol@cnrs.fr, est chargé de recherche au Centre National de la Recherche Scientifique, membre de l'UMR PRODIG (Paris). 